\title{
MicroRNA expression patterns in the malignant progression of gliomas and a 5-microRNA signature for prognosis
}

\author{
Wei Yan ${ }^{1,2, *}$, Rui Li ${ }^{2, *}$, Yanwei Liu1,4,*, Pei Yang ${ }^{1,4}$, Zheng Wang1,4, Chuanbao Zhang ${ }^{1,4}$, \\ Zhaoshi Bao ${ }^{1,4}$, Wei Zhang ${ }^{1,4}$, Yongping You ${ }^{2}$ and Tao Jiang ${ }^{1,3,4}$ \\ ${ }^{1}$ Beijing Neurosurgical Institute, Capital Medical University, Beijing, PR China \\ ${ }^{2}$ Department of Neurosurgery, First Affiliated Hospital of Nanjing Medical University, Nanjing, PR China \\ ${ }^{3}$ Beijing Institute for Brain Disorders Brain Tumor Center, Beijing, PR China \\ ${ }^{4}$ Department of Neurosurgery, Beijing Tiantan Hospital, Capital Medical University, Beijing, PR China \\ * These authors contributed equally to this work \\ Correspondence to: Tao Jiang, email: taojiang1964@163.com
}

Yongping You, email: yypl9@njmu.edu.cn

Keywords: Anaplastic Gliomas; Secondary Glioblastomas; Proneural Glioblastomas; MicroRNAs; MiR-105-767 cluster; TCGA

Received: August 24, 2014

Accepted: November 04, 2014

Published: November 04, 2014

This is an open-access article distributed under the terms of the Creative Commons Attribution License, which permits unrestricted use, distribution, and reproduction in any medium, provided the original author and source are credited.

\section{ABSTRACT}

MicroRNAs (miRNAs) are directly involved in the progression in various cancers. To date, no systematic researches have been performed on the expression pattern of miRNA during progression from low grade gliomas to anaplastic gliomas or secondary glioblastomas and those prognostic miRNAs in anaplastic gliomas and secondary glioblastomas. In the present study, high-throughput microarrays were used to measure miRNA expression levels in 116 samples in the different progression stages of glioma. We found that miRNA expression pattern totally altered when low grade gliomas progressed to anaplastic gliomas or secondary glioblastomas. However, anaplastic gliomas and secondary glioblastomas have similar expression pattern in miRNA level. Furthermore, we developed a five-miRNA signature (two protective miRNAs-miR-767-5p, miR-105; three risky miRNAs: miR-584, miR-296-5p and miR-196a) that could identify patients with a high risk of unfavorable outcome in anaplastic gliomas regardless of histology type. It should be highlighted that the five-miRNA signature can also identify patients who had a high risk of unfavorable outcome in secondary and TCGA Proneural glioblastomas, but not Neural, Classical and Mesenchymal glioblastomas. Taken together, our results demonstrate that miRNA expression patterns in the malignant progression of gliomas and a novel prognostic classifier, the five-miRNA signature, serve as a prognostic marker for patient risk stratification in anaplastic gliomas, Secondary and Proneural glioblastomas.

\section{INTRODUCTION}

MicroRNAs (miRNAs) belong to a recently discovered class of small, non-coding RNA molecules that regulate the expression of multiple target genes and multiple cellular processes including cell differentiation, stem cell maintenance, and epithelial-mesenchymal transition. $[1,2]$ The abnormal expression of miRNAs is a common feature of cancers and can be caused by different mechanisms such as amplification/deletion, chromosomal rearrangements, and epigenetic regulation.
$[3,4]$ Depending on the genes targeted, miRNAs can act as either oncogenes or tumor suppressors. [5-8] MiRNAs show characteristic expression signatures in various cancers and can profoundly affect cancer cell behavior $[9$, 10]. Low grade gliomas inherently tend to locally recur and spontaneously progress to anaplastic gliomas and eventually secondary glioblastomas.[11] However, to date, the miRNA expression patterns in the malignant progression of gliomas have not been investigated systematically.

In this study, we present the results of miRNA 
expression patterns from 116 glioma samples by microarray analysis. We found that miRNA expression pattern totally altered when low grade gliomas progressed to anaplastic gliomas or secondary glioblastomas. However, anaplastic gliomas and secondary glioblastomas have similar expression pattern in miRNA level. Meanwhile, survival analysis revealed that a five-miRNA signature (two protective miRNAs-miR-767-5p, miR105; three risky miRNAs: miR-584, miR-296-5p and miR-196a) that could identify patients with a high risk of unfavorable outcome in anaplastic gliomas, secondary and TCGA Proneural glioblastomas, but not Neural, Classical and Mesenchymal glioblastomas.

\section{RESULTS}

\section{Whole genome miRNA profiling reveals miRNA expression patterns in the malignant progression of gliomas}

In the present study, SAM was performed to compare the differential expressed miRNAs among low grade, anaplastic gliomas and secondary glioblastomas in a pairwise manner (Supplementary Figure 1). Only those miRNAs (SAM: Fold change $>1.5$, Q value $<1 \%$ ) were taken as significant miRNAs. Then samples were ordered from low grade glioma to secondary glioblastomas, and significant genes were clustered (Figure 1). As shown in Figure 1 and Supplementary Figure 1, the miRNA expression pattern of secondary glioblastomas resembles that of anaplastic gliomas very much. It is very possible that the main alteration of miRNAs occurred during malignant progression from low-grade glioma to anaplastic gliomas or secondary glioblastomas, but not anaplastic gliomas to secondary glioblastomas. In the present study, we observed aberrant expression of several well-characterized tumorigenesis-related miRNAs (such as miR-16, miR-17, miR-19a, miR-20a, miR-328, miR$181 \mathrm{a}$ and let-7 family members), which also showed the same increased or reduced expression upon progression, as well as a number of miRNAs whose function is currently unknown (such as miR-590-3p). In addition, we also validated the expression of miR-590-3p in an independent validation cohort [Supplementary Figure 2].

\section{Identification of the five-miRNA signature and its association with survival in anaplastic gliomas}

Following data filtering, described in the "Materials and Methods", the remaining 629 miRNAs were analyzed by BRB array tools using the permutation test method. We identified five miRNAs (miR-767-5p, miR-105, miR584, miR-296-5p and miR-196a) that are significantly associated with overall survival (Permutation $\mathrm{P}<0.01$; Figure 2). The significant miRNAs that formed the signature were of two types - risky and protective. Risky miRNAs were defined as those that had hazard ratio for death greater than 1. Protective miRNAs were defined as those that had hazard ratio for death less than 1 . Using this definition, we found 2 protective miRNAs and 3 risky miRNAs. Of note, 2 protective miRNAs: miR-105 and miR-767-5p are located in the same miRNA cluster. We then used the five miRNAs identified to construct a signature by the risk-score method.[15] As shown in Figure 3A-J, the five-miRNA signature risk score was calculated for each of the 44 patients in the Microarray cohort and then was used to divide them into a high-risk group and a low-risk group in all anaplastic gliomas, astrocytomas, oligodendrocytomas and oligoastrocytomas. Furthermore, the prognostic value of the five-miRNA signature was validated using real-time qRT-PCR in an independent cohort consisting of 134 samples (Figure 3KT).

\section{The five-miRNA signature predict the clinical outcome of secondary and TCGA Proneural glioblastomas}

In the present study, secondary glioblastomas have similar miRNA expression pattern with anaplastic gliomas. So we further evaluated whether the five-miRNA signature could identify patients with a high risk of unfavorable outcome in secondary glioblastomas. As shown in Figure 4, the five-miRNA signature was also associated with the prognosis of secondary glioblastomas.

To validate the five-miRNA signature in TCGA glioblastomas, paired miRNA and mRNA profiling data (level3) was downloaded from TCGA data portal. Total 491 TCGA glioblastoma samples were included in our study. We first annotated the TCGA glioblastoma samples with TCGA classification system based on mRNA expression. After removing the patients whose survival time were lesser than 30 days, Total 349 patients (77 Proneural, 65 Neural, 90 Classical and 117 Mesenchymal glioblastomas) who had survival information were for further analysis. As shown in Figure 5, the five-miRNA signature could identify patients with a high risk of unfavorable outcome in Proneural glioblastomas $(\mathrm{P}=$ 0.0043). However, the five-miRNA signature could not predict the clinical outcome in Neural, Classical and Mesenchymal glioblastomas $(\mathrm{P}=0.4185,0.265$, and 0.5311 , respectively).

\section{DISCUSSION}

MiRNAs show characteristic expression signatures in various cancers and can profoundly affect cancer cell behavior.[16] Low grade gliomas inherently tend to 


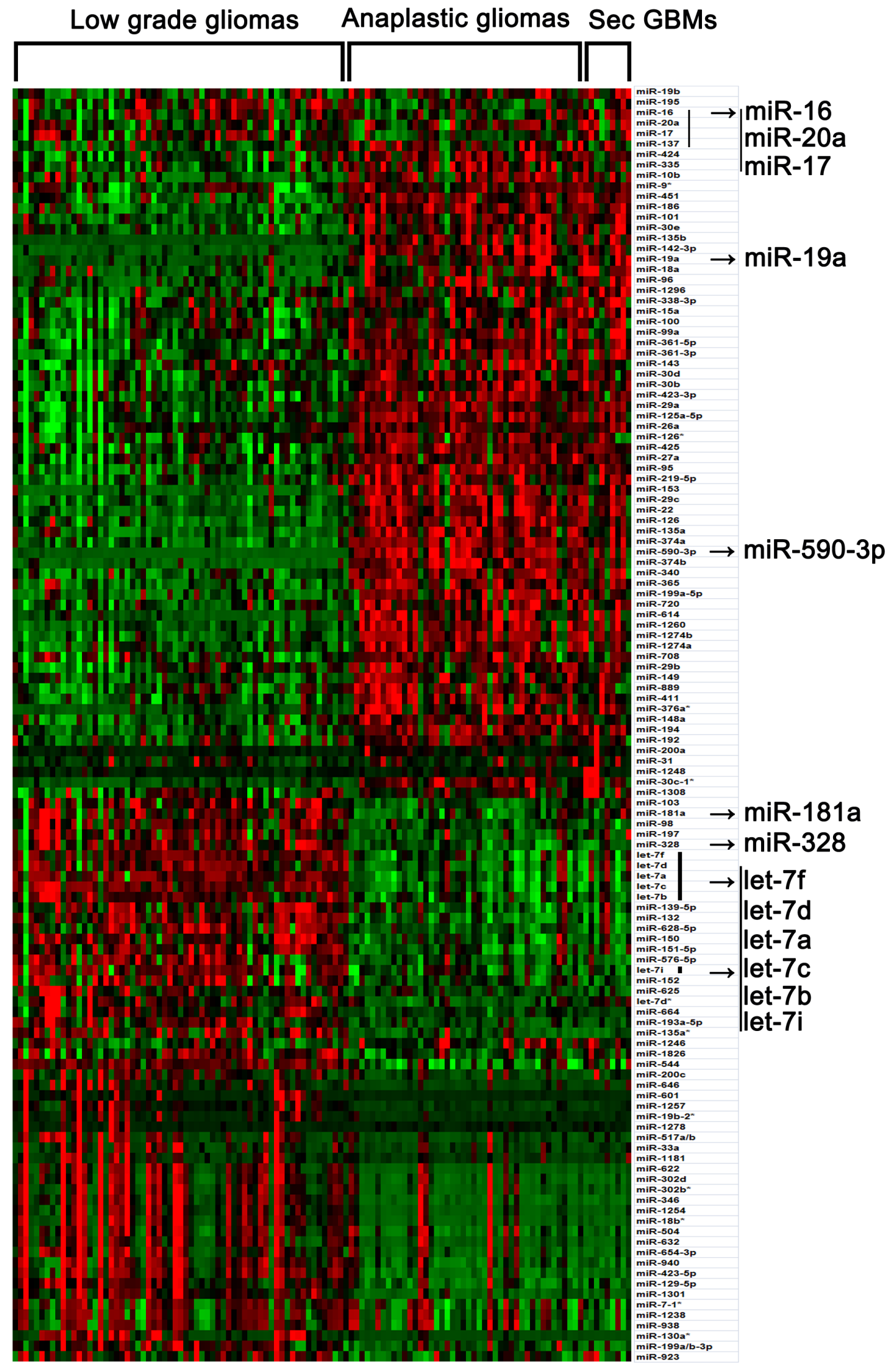

Figure 1: Differential expressed miRNAs among low grade, anaplastic gliomas and secondary glioblastomas (SAM: Fold change $>1.5, Q$ value $<1 \%$ ). Total 116 samples were ordered from low grade glioma to secondary glioblastomas, and differential expressed miRNAs were clustered. Arrows indicated genes that were discussed in the text. 


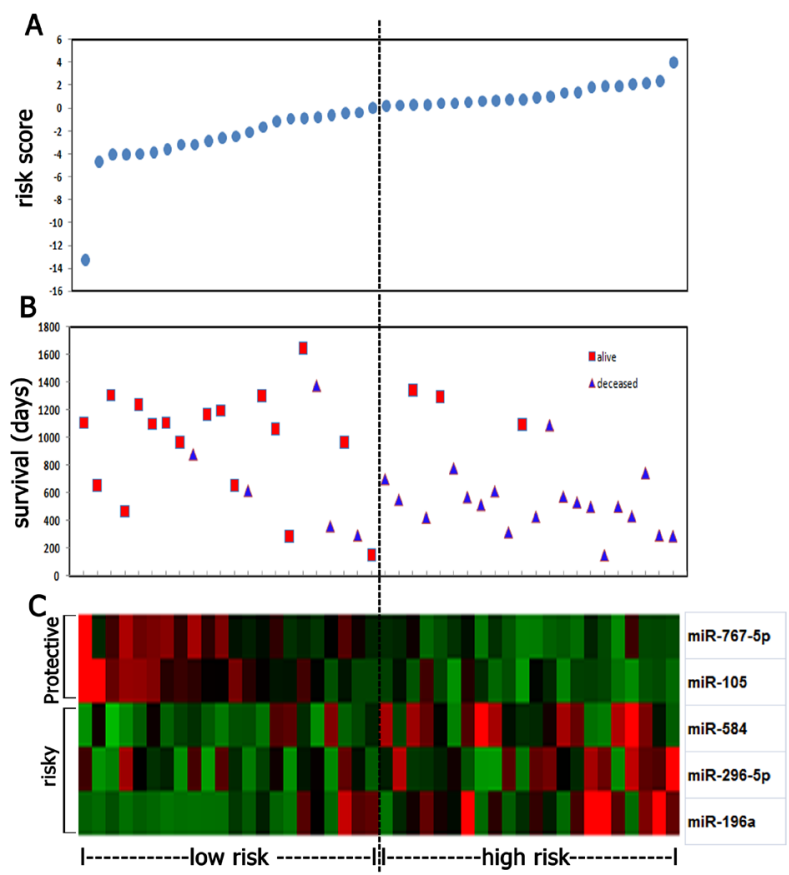

Figure 2: Five miRNA risk-score analysis of anaplastic gliomas $(n=44)$. (A) The prognostic miRNA signature risk-score distribution. (B) Patients' survival status and time. (C) Color-gram of miRNA expression profiles of GBM patients; rows represent risky and protective miRNAs, and columns represent patients. The black dotted line represents the miRNA signature cutoff dividing patients into low-risk and high-risk groups.
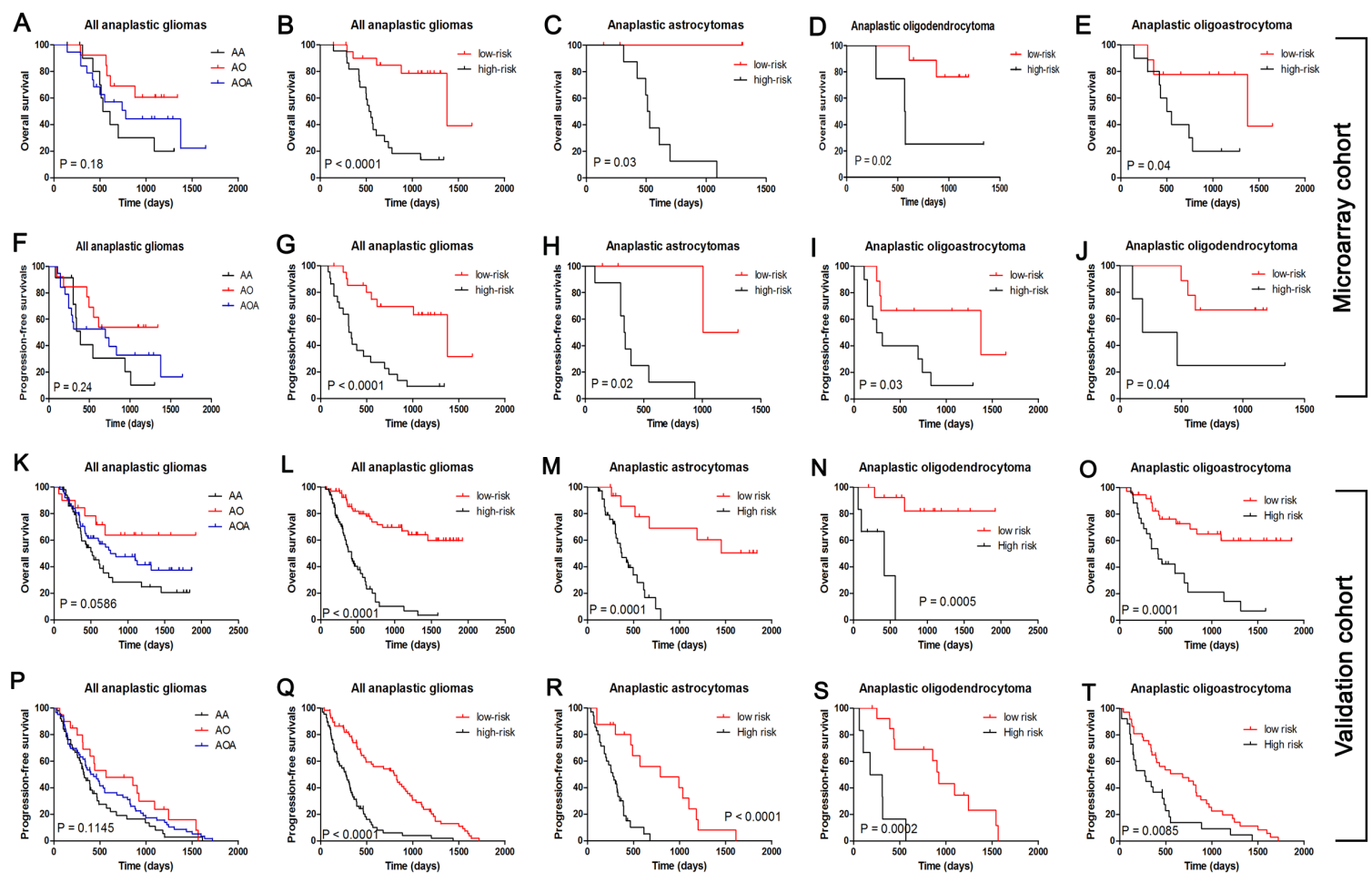

Histology stratification

The five-miRNA stratification

Figure 3: The five-miRNA signature was tightly associated with prognosis in both Microarray cohort $(n=44)$ and Validation cohort $(\mathbf{n}=\mathbf{1 3 4})$ independent of histology type. Histology types could stratify the anaplastic gliomas into different prognostic subpopulation although the $\mathrm{P}$ value is not enough small in both Microarray (A and F).and Validation cohort (K and P). The fivemiRNA signature was could stratify the all anaplastic gliomas into two distinct prognostic subpopulation in both Microarray (B and G).and Validation cohort (L and Q). In each histological subtype of anaplastic gliomas (astrocytomas, oligodendrocytomas and oligoastrocytomas), the five-miRNA signature could also be taken as a stable method used for prognosis stratification in both Microarray (C, D, E, H, I and J) and Validation cohort (M, N, O, R, S and T). 
A

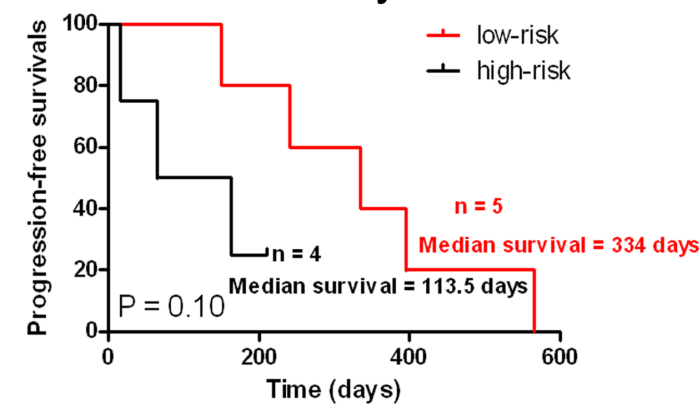

C

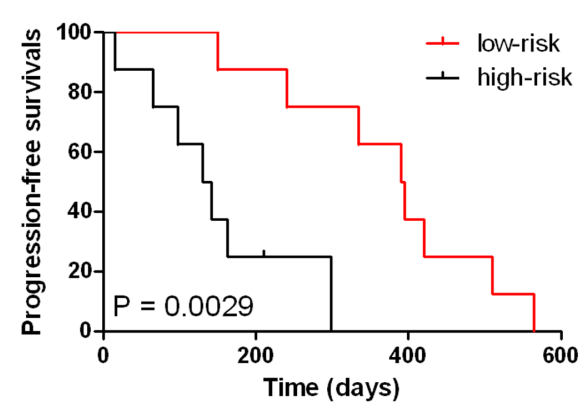

B

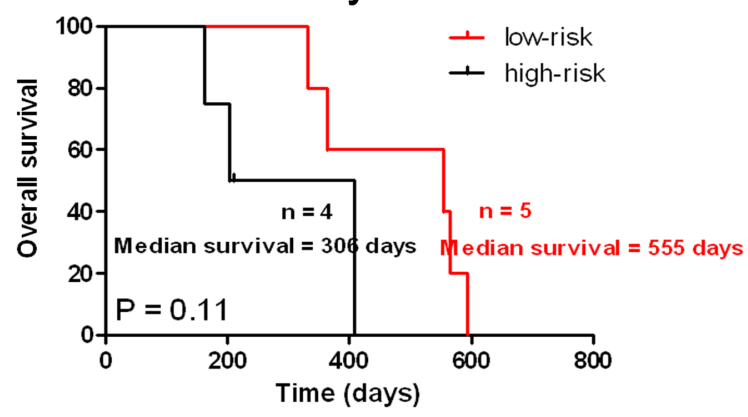

D

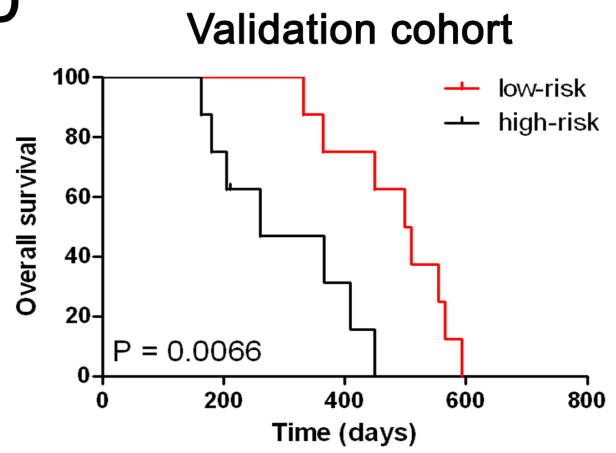

Figure 4: The five-miRNA signature was tightly associated with prognosis in secondary glioblastomas. The five-miRNA signature was could stratify the all secondary glioblastomas into two distinct prognostic subpopulation in both Microarray (A and B).and Validation cohort (C and D).

A

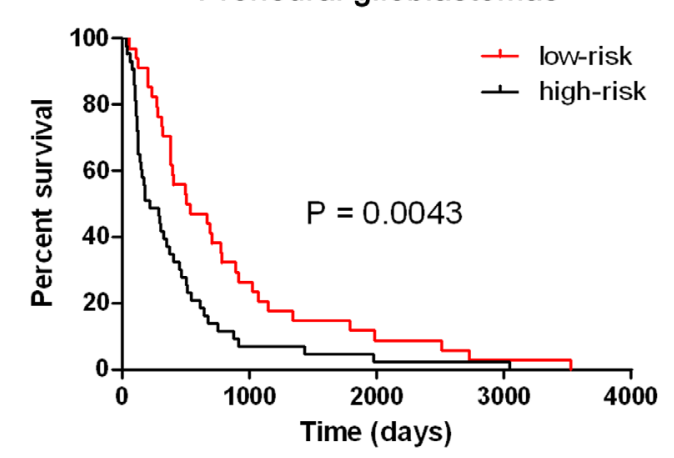

C

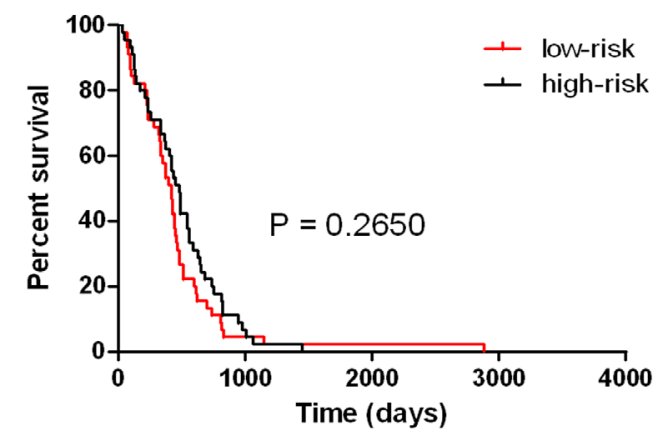

B

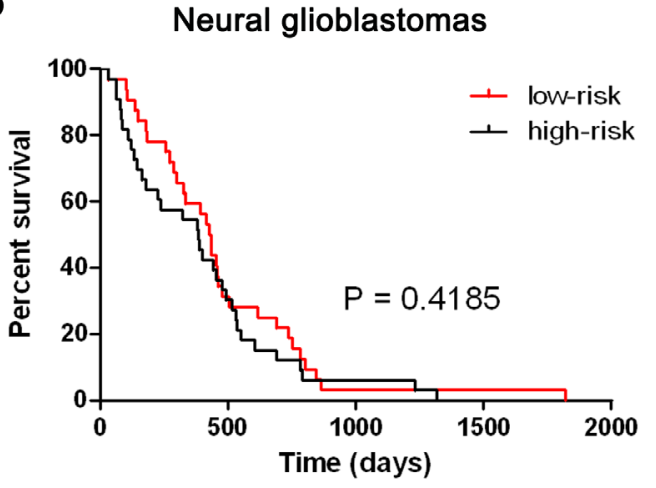

D

Mesenchymal glioblastomas

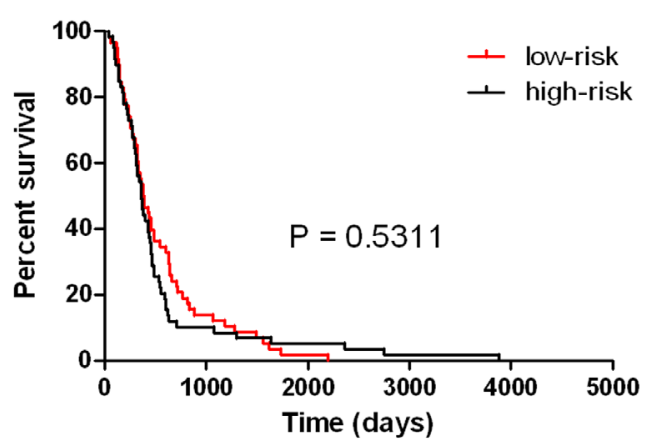

Figure 5: The five-miRNA signature could predict the clinical outcome of TCGA Proneural glioblastomas. The fivemiRNA signature was could stratify TCGA Proneural glioblastomas into two distinct prognostic subpopulation (A). However, the fivemiRNA signature could not predict the clinical outcome in Neural, Classical and Mesenchymal glioblastomas (B, C and D). 
locally recur and spontaneously progress to anaplastic gliomas and eventually secondary glioblastomas.[11] However, to date, the miRNA expression patterns in the malignant progression of gliomas have not been investigated systematically. In the present study, we analyzed the whole-genome miRNA profiles 116 samples in the different progression stages of glioma and found that miRNA expression pattern totally altered when low grade gliomas progressed to anaplastic gliomas or secondary glioblastomas. However, anaplastic gliomas and secondary glioblastomas have similar expression pattern in miRNA level. In addition, several progression associated miRNAs in previous reports (miR-16, miR-17, miR-19a, miR-20a, miR-328, miR-181a and let-7 family members) also showed the same increased or reduced expression upon progression in our study.[11, 17] Our observations indicated that miRNA may play a critical role during progression from low grade gliomas to anaplastic gliomas or secondary glioblastomas and not contribute to the malignant progression from anaplastic gliomas to secondary glioblastomas.

Anaplastic gliomas are classified by the WHO as grade 3 malignant tumors and include anaplastic astrocytomas, anaplastic oligodendrogliomas and anaplastic oligoastrocytomas.[18] It has previously been shown that miRNA expression patterns could be used as prognostic indicators in various cancers. $[19,20]$ Recently, Srinivasan et al. reported that a ten-miRNA expression signature predicts survival in glioblastoma. [15] However, to date, no prognostic markers based on miRNA profiling have been identified in anaplastic gliomas. In the present study, we developed a five-miRNA signature (two protective miRNAs-miR-767-5p, miR105; three risky miRNAs: miR-584, miR-296-5p and miR-196a) that could identify patients with a high risk of unfavorable outcome in anaplastic gliomas regardless of histology type. Of them, only miR-196a was previously reported to be negatively correlated with overall survival in glioblastomas.[21] It should be highlighted that two protective miRNAs (miR-767-5p and miR-105) localized in the same miRNA cluster. Our observations showed a novel prognostic classifier, the five-miRNA signature, in anaplastic gliomas and revealed an important role for the miR-105-767 cluster in the pathogenesis and tumor biology of anaplastic gliomas for the first time. In addition, we applied the five-miRNA signature in secondary and TCGA glioblastomas. We found that the five-miRNA signature can also identify patients who had a high risk of unfavorable outcome in secondary and TCGA Proneural glioblastomas, but not Neural, Classical and Mesenchymal glioblastomas. The molecular and clinical characteristics of Proneural samples in primary glioblastomas resemble secondary glioblastoma and may progress from lower grade gliomas thought without clinical or histological evidence of a less malignant precursor lesion. Thus, we postulated that Proneural glioblastomas might have similar
miRNA expression pattern with anaplastic gliomas and secondary glioblastomas and undergo potential malignant progression from low grade gliomas without a previous operation.

In summary, our results demonstrate that miRNA may play a critical role during progression from low grade gliomas to anaplastic gliomas or secondary glioblastomas and not contribute to the malignant progression from anaplastic gliomas to secondary glioblastomas. We further provide a novel prognostic classifier, the fivemiRNA signature, serve as a prognostic marker for patient risk stratification in anaplastic gliomas, Secondary and Proneural glioblastomas.

\section{PATIENTS AND METHODS}

\section{Patients and samples}

All glioma samples included in our study were from the Chinese Glioma Genome Atlas (CGGA). The patients underwent surgical resection between January 2006 and December 2009. Patients were eligible for the study if the diagnosis of glioma was established histologically according to the $2007 \mathrm{WHO}$ classification. Tumor tissue samples were obtained by surgical resection before treatment with radiation and chemotherapy. This study was approved by the institutional review boards of the hospitals, and written informed consent was obtained from all patients.

\section{RNA extraction, Whole genome miRNA profiling and Real-time qRT-PCR}

RNA extraction, Whole genome miRNA profiling and Real-time qRT-PCR were performed as Ref.[12] Briefly, total RNA from frozen tumor tissues was extracted using the mirVana miRNA Isolation kit (Ambion, Austin, TX, USA) according to the manufacturer's protocol. Then, $200 \mathrm{ng}$ of total RNA was polyadenylated and then converted into cDNA using a biotin-labeled Oligo dT primer with a universal PCR sequence. After cDNA synthesis, miRNA were individually interrogated using specific oligonucleotides. A single miRNA-specific Oligo (MSO) was designed against each mature miRNA sequence, and miRNA-specific primers were extended using DNA polymerase. Universal primers were used to amplify the cDNA templates, and the primer complimentary to the array was fluorescently labeled. Finally, the labeled, single-stranded PCR products were hybridized to the Human v2.0 miRNA Expression BeadChip (Illumina, Inc., San Diego, CA, USA) containing 1,146 human miRNAs ( $97 \%$ coverage of the miRBase 12.0 database). Real-time qRT-PCR was performed using a standard TaqMan PCR kit procedure on 
a LightCycler 480 real-time PCR system (Roche). All of the primers and probes for the TaqMan microRNA assays were purchased from Applied Biosystems. Real-time RT-PCR was carried out according to the manufacturer's recommendation, and the relative expression was calculated using the comparative $\mathrm{Ct}$ method.

\section{Data filtering and statistical analysis}

Median absolute deviation (MAD) was calculated using MATLAB software. After filtering the probes showing the lowest variable expression (MAD < 0.05), the remaining 629 miRNAs were used for the following analysis. We applied significance analysis of microarrays (SAM) to identify differential expressed miRNAs in low grade, anaplastic gliomas and secondary glioblastomas in a pairwise manner. Student's t-test was used to determine significant differences. Cox proportional hazard regression analyses were performed using the BRB array tools in the Microarray cohort. Using the permutation test method, with 10000 permutations, we found 5 miRNAs were strongly correlated with survival $(p<0.01)$. The significant miRNAs were divided into risky and protective types. Risky miRNAs were defined as miRNAs with a hazard ratio for death greater than 1 . In contrast, protective miRNAs were defined based on a hazard ratio for death less than 1. Using these 5 significant miRNAs, a risk-score formula for survival prediction was constructed according to a linear combination of the expression level of the miRNA, weighted by the regression coefficient from the univariate Cox regression analyses.[13-15] According to this model, patients having high risk scores are expected to have poor survival outcomes as compared to patients having low risk scores. The risk scores are calculated as follows: Risk score $=(-5.04 \mathrm{x}$ expression of hsa-miR$767-5 p)+(6.5 x$ expression of hsa-miR-296-5p) $+(0.76$ $\mathrm{x}$ expression of hsa-miR-584) $+(-3.14 \mathrm{x}$ expression of hsa-miR-105) $+(0.9$ x expression of hsa-miR-196a). We used the 50th percentile risk score as the cut-off, since this divided the Microarray samples into two groups having different survival times with highest significance. KaplanMeier survival analysis was used to estimate the survival distributions, and the log-rank test was used to assess the statistical significance between stratified survival groups using GraphPad Prism 5.0 statistical software. Prediction analysis of microarrays was used to subtype annotation. All data are presented as the means \pm SE. A two-sided $\mathrm{P}$ value of $<0.05$ was regarded as significant.

\section{ACKNOWLEDGMENTS}

This work was supported by grants from the National High Technology Research and Development Program of China (863) (No.2012AA02A508), International Cooperation Program (No. 2012DFA30470),
National Natural Science Foundation of China (No. 81201993), National Natural Science Foundation of China (No.81101901), Jiangsu Province's Key Provincial Talents Program (No.RC2011051) and Jiangsu Province's Key Discipline of Medicine (No.XK201117).

\section{Conflict of interest}

None of the authors has any conflict of interest to disclose.

\section{REFERENCES}

1. Schickel R, Boyerinas B, Park SM and Peter ME. MicroRNAs: key players in the immune system, differentiation, tumorigenesis and cell death. Oncogene. 2008; 27(45):5959-5974.

2. Lee HK, Finniss S, Cazacu S, Bucris E, Ziv-Av A, Xiang C, Bobbitt K, Rempel SA, Hasselbach L, Mikkelsen T, Slavin S and Brodie C. Mesenchymal stem cells deliver synthetic microRNA mimics to glioma cells and glioma stem cells and inhibit their cell migration and self-renewal. Oncotarget. 2013; 4(2):346-361.

3. Calin GA and Croce CM. MicroRNAs and chromosomal abnormalities in cancer cells. Oncogene. 2006; 25(46):62026210.

4. Asuthkar S, Velpula KK, Chetty C, Gorantla B and Rao JS. Epigenetic regulation of miRNA-211 by MMP-9 governs glioma cell apoptosis, chemosensitivity and radiosensitivity. Oncotarget. 2012; 3(11):1439-1454.

5. Wang D, Qiu C, Zhang H, Wang J, Cui Q and Yin Y. Human microRNA oncogenes and tumor suppressors show significantly different biological patterns: from functions to targets. PLoS One. 2010; 5(9).

6. Speranza MC, Frattini V, Pisati F, Kapetis D, Porrati P, Eoli M, Pellegatta S and Finocchiaro G. NEDD9, a novel target of miR-145, increases the invasiveness of glioblastoma. Oncotarget. 2012; 3(7):723-734.

7. Smits M, Nilsson J, Mir SE, van der Stoop PM, Hulleman E, Niers JM, de Witt Hamer PC, Marquez VE, Cloos J, Krichevsky AM, Noske DP, Tannous BA and Wurdinger T. miR-101 is down-regulated in glioblastoma resulting in EZH2-induced proliferation, migration, and angiogenesis. Oncotarget. 2010; 1(8):710-720.

8. Li H and Yang BB. Stress response of glioblastoma cells mediated by miR-17-5p targeting PTEN and the passenger strand miR-17-3p targeting MDM2. Oncotarget. 2012; 3(12):1653-1668.

9. Wang L, Shi ZM, Jiang CF, Liu X, Chen QD, Qian X, Li DM, Ge X, Wang XF, Liu LZ, You YP, Liu N and Jiang BH. MiR-143 acts as a tumor suppressor by targeting N-RAS and enhances temozolomide-induced apoptosis in glioma. Oncotarget. 2014; 5(14):5416-5427.

10. Bier A, Giladi N, Kronfeld N, Lee HK, Cazacu S, 
Finniss S, Xiang C, Poisson L, deCarvalho AC, Slavin S, Jacoby E, Yalon M, Toren A, Mikkelsen T and Brodie C. MicroRNA-137 is downregulated in glioblastoma and inhibits the stemness of glioma stem cells by targeting RTVP-1. Oncotarget. 2013; 4(5):665-676.

11. Malzkorn B, Wolter M, Liesenberg F, Grzendowski M, Stuhler K, Meyer HE and Reifenberger G. Identification and functional characterization of microRNAs involved in the malignant progression of gliomas. Brain Pathol. 2010; 20(3):539-550.

12. Yan W, Zhang W, You G, Zhang J, Han L, Bao Z, Wang Y, Liu Y, Jiang C, Kang C, You Y and Jiang T. Molecular classification of gliomas based on whole genome gene expression: a systematic report of 225 samples from the Chinese Glioma Cooperative Group. Neuro Oncol. 2012; 14(12):1432-1440.

13. Dave SS, Wright G, Tan B, Rosenwald A, Gascoyne RD, Chan WC, Fisher RI, Braziel RM, Rimsza LM, Grogan TM, Miller TP, LeBlanc M, Greiner TC, Weisenburger DD, Lynch JC, Vose J, et al. Prediction of survival in follicular lymphoma based on molecular features of tumor-infiltrating immune cells. N Engl J Med. 2004; 351(21):2159-2169.

14. Zhao Q and Sun J. Cox survival analysis of microarray gene expression data using correlation principal component regression. Stat Appl Genet Mol Biol. 2007; 6:Article16.

15. Srinivasan S, Patric IR and Somasundaram K. A tenmicroRNA expression signature predicts survival in glioblastoma. PLoS One. 2011; 6(3):e17438.

16. Godlewski J, Nowicki MO, Bronisz A, Williams S, Otsuki A, Nuovo G, Raychaudhury A, Newton HB, Chiocca EA and Lawler S. Targeting of the Bmi-1 oncogene/ stem cell renewal factor by microRNA-128 inhibits glioma proliferation and self-renewal. Cancer Res. 2008; 68(22):9125-9130.

17. Shi L, Cheng Z, Zhang J, Li R, Zhao P, Fu Z and You Y. hsa-mir-181a and hsa-mir-181b function as tumor suppressors in human glioma cells. Brain Res. 2008; 1236:185-193.

18. DeAngelis LM. Anaplastic glioma: how to prognosticate outcome and choose a treatment strategy. [corrected]. J Clin Oncol. 2009; 27(35):5861-5862.

19. Tan X, Qin W, Zhang L, Hang J, Li B, Zhang C, Wan J, Zhou F, Shao K, Sun Y, Wu J, Zhang X, Qiu B, Li N, Shi S, Feng $\mathrm{X}$, et al. A 5-microRNA signature for lung squamous cell carcinoma diagnosis and hsa-miR-31 for prognosis. Clin Cancer Res. 2011; 17(21):6802-6811.

20. Schaefer A, Jung M, Mollenkopf HJ, Wagner I, Stephan C, Jentzmik F, Miller K, Lein M, Kristiansen G and Jung K. Diagnostic and prognostic implications of microRNA profiling in prostate carcinoma. Int J Cancer. 2010; 126(5):1166-1176.

21. Guan Y, Mizoguchi M, Yoshimoto K, Hata N, Shono T, Suzuki SO, Araki Y, Kuga D, Nakamizo A, Amano T, Ma X, Hayashi K and Sasaki T. MiRNA-196 is upregulated in glioblastoma but not in anaplastic astrocytoma and has prognostic significance. Clin Cancer Res. 2010; 16(16):4289-4297. 